\title{
A Scoring Rule-based Truthful Demand Response Mechanism
}

\author{
Keisuke Hara $^{1}$ and Takayuki Ito ${ }^{1}$ \\ ${ }^{1}$ Department of Techno-Business Administration, \\ Department of Computer Science, \\ Nagoya Institute of Technology \\ Gokiso, Showa-ku, Nagoya 466-8555, Japan. \\ E-mail: ito.takayuki@nitech.ac.jp
}

\begin{abstract}
Demand Response (DR) has been extensively studied as one of the important features of smart grid. The DR strategies can be grouped into two categories, one is incentive-based DR and the other is pricing-based DR. Our work focuses on DR involving both pricing factor and incentive factor using scoring rule. In the literature, several DR mechanisms have been proposed, however, most studies have not focused on the cooperation among consumers although it is important to devise an efficient and stable DR. In this paper, we propose a cooperative demand response mechanism by using a truthful allocation mechanism with scoring rule. The brief ideas of our model are the following: the consumers will be rewarded a discount on the price to measure up how well they predict demand shift. A reward mechanism is based on a strictly proper scoring rule. This mechanism is applied between consumer agents (CA) to Cooperative Demand Response System (CDRS) and Generation Company (GENCO). The proposed mechanism is tested on real data provided by Chubu Electric Power Company and we show that this mechanism is capable of reducing peak demand.
\end{abstract}

Keywords: Mechanim Design, Scoring Rule, Demand Response

\section{Introduction}

With the growing needs of environmental sustainability and continuous change in electric power deregulation, smart grid becomes an inevitable choice for the society. As one of the important features of smart grid is Demand Response (DR). DR is gaining importance in designing grid functionalities specially at the end user (consumers) level. Formally speaking, DR is a mechanism that influences the consumers to modify their energy usages from the normal consumption patterns in response to the changes in the price of electricity over time ${ }^{2}$.

In order to fully utilize the DR capability, smart houses had already start to adopt devices which can be controlled, maintained, monitored and even scheduled as necessary. Smart house technologies make all electronic devices around the house to act "smart" and become more autonomous. Most of the important appliances in the future will take advantage of this technology through home networks and the Internet. Such feature of smart grid is a way for ordinary electronics and appliances to communicate among themselves, consumers and even higher entities such as GENCOs. In such an environment, consumers (actually a consumer agent, refereed as CA hereafter, will be responsible to take such decision in conjunction with smart-meter) can respond to day-ahead dynamic pricing signal effectively and also intelligently managing and scheduling devices thereby flattening out peak demand and achieving better resource utilization. 
DR has been studied in various fields. DR strategies can be grouped into two general categories, one is pricing-based DR and another is incentive based DR. In the pricing-based DR ${ }^{21,26}$, consumers dynamically adjust their consumption according to the time varying pricing while maximizing their payoffs. And for incentive based DR ${ }^{8,6}$, consumers are given incentives in payment, to reduce their consumption in response to the system reliability. Pricing-based DR such as RTP (Real-Time Pricing) has problem in terms of an efficient stable power system operation, because it is too difficult to know in advance how much consumers actually participate in DR. As an example, ${ }^{1}$ shows that RTP mechanisms do not necessarily lead to peak-to-average ratio reduction, because large portions of load may be shifted from a typical peak hour to a typical nonpeak hour. When attention is paid only to efficiency and stability, "direct load control" in which a power company controls the amount of electricity consumption of consumers from outside is most effective, but it has problem in terms of usability. In addition, most studies have not focused on the cooperation among consumers, but it is important to develop efficient and stable DR.

On a different note, in order to numerically measure the actual realization of a probabilistic event which will forecast ahead, scoring rule was defined 15,4 . Moreover, it binds the assessor to make a careful prediction and hence truthfully elicit his/her private preferences. That is why, scoring rule has been applied successfully while designing a truthful incentive mechanism in a diverse applications such as voting rules 3119 . Strictly proper scoring rules can be employed by a mechanism designer to ascertain that agents accurately declare their privately calculated distributions, reflecting their confidence in their own forecast. The applicability of scoring rule is being investigated in field of smart-grid. For instance, ${ }^{24}$ presented a methodology for predicting aggregated demand in smart grid.

However, it is not enough to apply scoring rule to our model because our model uses auction for task allocation. When using scoring rule alone, agents are in fact able to misreport their belief in order to get task. Therefore, we also apply Viclrey-Clarke-
Groves Mechanism ${ }^{30,9,16}$ to our model. The applicability of VCG mechanism is being investigated in field of demand side management. For instance, 25 presented a VCG mechanism for demand side management programs to encourage efficient energy consumption among the users. In our model, we combine scoring rule and VCG mechanism to truthfully elicit agent's private preferences.

This paper presents a scoring rule based truthful cooperative demand response mechanism for CAs provided by the GENCO in response to the dynamic day-ahead time dependent pricing. The proposed method can be viewed as a bridge between incentive based DR and pricing based DR. The main ideas of our model can be summarized as follows: the consumers will be rewarded a discount on the price to measure how well they predict the shift demand that represents shifting the devices/loads towards specified time periods. The reward mechanism is based on a strictly proper scoring rule and VCG mechanism. The scoring rule is chosen to work with continuous variable (the normal distribution, as in the proposed method) and measure how accurate the prediction could be. The Continuous Ranked Probability Score ${ }^{15}$ possess such characteristics. In our mechanism, CA has incentive to participate in CDR using The Wisdom of Crowds ${ }^{27}$ such as lot of CAs prediction is better than a CA prediction. In addition, our mechanism has several desirable properties such as truthfulness and individual rationality and scalability.

The contributions of this paper are summarized as follows:

- We propose a scoring rule based truthful cooperative demand response mechanism for GENCO to encourage efficient peak cut. The proposed model can be viewed as a bridge between incentive based DR and pricing based DR. The consumers will be rewarded a discount on the price to measure up how well they predict the shift demand.

- We investigate some of the desired properties of our model. Such as, truthfulness and individualrationality of the proposed mechanism are proved and we fulfill those properties by combining scoring rule and VCG mechanism.

- We show that, each consumer has incentive to 
cooperate with each other by comparing CDRS and Singleton in experimental results. In addition, proposed model has scalability that the even-though number of CA increases, Wisdom of Crowd ${ }^{27}$ can work and the accuracy of prediction of CDRS also increase. We show that the larger the number of CA, gives better accuracy of prediction in the experiment.

- We tested proposed mechanism on real data provided by Chubu Electric Power Company and we validated that, this mechanism is capable to reduce peak demand.

The remainder of the paper is organized as follows, Firstly, we describe our model of cooperative demand response. Second, we describe the details of CDR Algorithms and a mechanism. Third, we describe the results of the experiments and our evaluation. Fourth, we describe the relative works. Finally, we conclude with a discussion of possible avenues for future work.

\section{Cooperative Demand Response Model}

We begin the situation where $N$ consumer agents (CAs) use a same generation company (GENCO). In this paper, we represent cooperation among CAs introducing CDRS (Cooperative Demand Response System) that represents a set of CAs. Each CA has a smart meter that communicates with the various devices at the CA and also GENCO. Each CA has two types of devices, one is "base load" that can not be shifted, such as lights or computers. Another is "flexible load" that can be shifted, such as AI robots or household electrical appliances. GENCO will try to flatten out the peak demand by incentivizing CA to cooperative shift flexible load consumption. CA will try to maximize the reward from GENCO. The reward depends on actual shift demand and accuracy of prediction.

Our model assumes a dynamic "day-ahead" pricing signal ${ }^{17}$, CAs receive their prices one day in advance. This pricing signal offers users more certainty than other common implementations of dynamic pricing 21,18 , such as "hour-ahead" or realtime pricing. With day-ahead pricing, CAs can schedule their device usage for the upcoming day so as to optimize their amount spent and willingness to shift their device usage. Hour-ahead or real-time pricing would force the ECC (Energy Consumption Controller) to use less optimal scheduling algorithm to solve an online knapsack problem. We note, however, that our algorithm can be easily adapted to hour-ahead pricing.

As a mechanism is design to incentivize CAs for providing private probabilistic information accurately (truthfully) and to the best of their forecasting ability, scoring rule is being applied in this model. Especially, strictly proper scoring rules can be employed by a mechanism designer to ascertain that agents accurately declare their privately calculated distributions, reflecting their confidence in their own forecast. The detailed flow of information and task assignment process are presented in Figure 1. As we can see, GENCO will send the price information as a signal to CAs. The price signal is typically determined based on the generation costs of electricity.

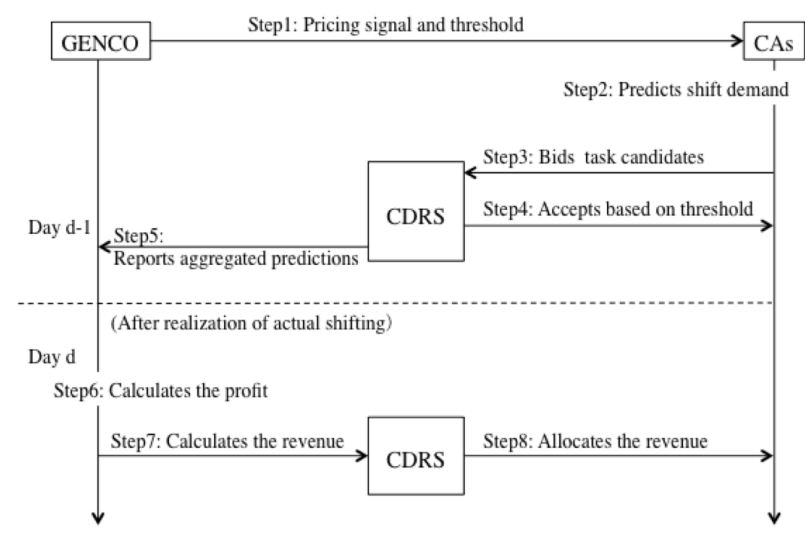

Fig. 1. CDR model flow

CA located in consumer's household integrated with ECC. Therefore, it can access the local information and data of that particular consumer. This information includes device usage schedule, duration, energy consumption, etc. CA also keeps track of the previous schedule prediction. Using such information plus the day-ahead dynamic pricing, CA makes a pre-schedule plan of different devices based on its forecasting accuracy and consumer's preferences. However, it will report CDRS the prediction confidence in a form of Gaussian distribution and tentative schedule of assigned devices. For each de- 
vice, CA calculates its uncertainty over the error it expects to make using a statical model of random errors. So, CA makes its prediction through a Gaussian Distribution. This assumption is based on the sampling of higher number of devices, since eventually an CDRS must handle a wide range of devices. Central Limit Theorem tells us in a case of wide range of events, the probability distribution that describes the sum of random variables tends towards a Gaussian Distribution as the sum approaches towards infinity. According to that, we can say that, in the perspective of CDRS, reporting error in the form of Gaussian Distribution is valid and portrays some form of accuracy.

We assume that each CA is various environment such as communication speed and load composition. Therefore, our model achieves the cooperation by combining scoring rule and VCG. Our model has some of the desired properties such as truthfulness and individual rationality and scalability based on The Wisdom of Crowds such as a lot of CAs prediction is better than a CA prediction.

\section{Algorithm}

In this section, we introduce detail of the CDR algorithm. First of all, set of CAs can be represented as $N=\{1,2, \ldots n\}$ and CAs try to maximise the expectation utility. Each CA has type $\theta_{i} \in \Theta_{i}$ which determines the preferences over different outcomes; i.e $v_{i}\left(a, \theta_{i}\right)$ is the value of CA $i$ with type $\theta_{i}$ for outcome $a \in A$. In our model, we use VCG mechanism and the choice rule $g: \Theta \rightarrow A$ and payment rule $p: \Theta \rightarrow \mathbb{R}$ and utility function are presented in Eq.(1) and Eq.(2) and Eq.(3).

$$
\begin{gathered}
g(\theta)=\underset{a \in A}{\arg \max } \sum_{i} v_{i}\left(a, \theta_{i}\right) \\
p_{i}(\theta)=\sum_{j \neq i} v_{j}\left(g\left(\theta_{-i}\right), \theta_{j}\right)-\sum_{j \neq i} v_{j}\left(g(\theta), \theta_{j}\right) \\
u_{i}\left(\theta_{i}\right)=v_{i}\left(a, \theta_{i}\right)-p_{i}(\theta)
\end{gathered}
$$

\section{Step1: Pricing signal and threshold}

GENCO estimates the total demand curve in the next day and assumes threshold $T h$ that signifies the goal of peak cut. In general, time periods that has larger demand is assigned high price, In order to shift and control demand ${ }^{3}$. In our model, we assume that there exist exactly two different price levels $_{\text {price }}>$ price $_{l}$. Time interval $t$ of each is expressed in Eq.(4).

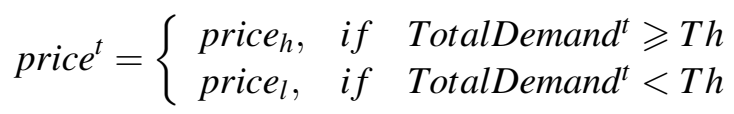

The intervals during which price $^{t}=$ price $^{h}$ are considered to be peak-intervals, at which consumption needs to be reduced.

\section{Step2: Predicts shift demand}

CAs predict the shift demand $s d_{i, t}^{p}$ based on price signal and load composition. CAs have two type of devices, one is "base load" and another is "flexible load". CAs predict the demand of flexible load in $t_{h}$. Therefore, predicted shift demand of CA $i$ in specific time periods $t$ is presented in Eq.(5). where $D V_{i}$ is a set of flexible load of $C A_{i}$.

$$
s d_{i, t}^{p}=\sum_{j \in D V_{i}} \text { ShiftDevicesDemand } d_{j, t}^{p}
$$

In case CAs do not behave as predicted, it may lead to retard in planned production of GENCO and decrease the peak-to-average ratio. In this model, we introduce a scoring rule to incentivize CAs for providing private probabilistic information truthfully. GENCO calculates the reward for CAs based on prediction of task achievement and prediction of confidence. Our model is dealing with devising a scoring rule for multi-item prediction. Therefore, conventional scoring rule with single item, such as Brier score ${ }^{5}$ is of no use for this mode. In order to rightfully incentivize the CA to make their prediction of device shifting for multiple items rightfully; the continuous ranked probability score (CRPS) is applied ${ }^{15}$. CRPS is a strictly proper scoring rule that is used for continuous variables since the traditional 
forms of proper and strictly proper scoring rules usually do not work with continuous variables. In the proposed method, Gaussian Distribution is used to model the consumer's device shifting prediction and associated confidence. The usage of CRPS is investigated before in distributed power system operation to rightfully score the distributed energy resources 23. CRPS is able to measure the closeness of the prediction that means how close a prediction to realized event where higher value is assigned to close prediction. The relative prediction error $e_{i, t}$ of $C A_{i}$ in each time periods as presented in Eq.(6).

$$
e_{i, t}=\frac{s d_{i, t}^{a}-s d_{i, t}^{p}}{s d_{i, t}^{p}}
$$

where $s d_{i, t}^{a}$ shows the actual shift demand. Lets assume each CA $i$ reports its relative prediction error in a form of uncertainty over it, represented by Gaussian Distribution Function $N\left(\mu=0, \sigma_{i, t}^{2}\right)$. Let $\sigma_{i, t}$ be $C A_{i}$ confidence of prediction, CRPS is presented in Eq.(7).

$$
\begin{aligned}
& \operatorname{CRPS}\left(N\left(\mu=0, \sigma_{i, t}^{2}\right), e_{i, t}\right) \\
& =\sigma_{i, t}\left[\frac{1}{\pi}-2 \varphi\left(\frac{e_{i, t}}{\sigma_{i, t}}\right)-\frac{e_{i, t}}{\sigma_{i, t}}\left(2 \Phi\left(\frac{e_{i, t}}{\sigma_{i, t}}\right)-1\right)\right]
\end{aligned}
$$

where the probability density function and cumulative distribution function for Gaussian Distribution Function are denoted as $\varphi$ and $\Phi$, respectively. The notation $\operatorname{CRPS}\left(N\left(\mu=0, \sigma_{i, t}^{2}\right), e_{i, t}\right)$ can be simplified using $\operatorname{CRPS}_{i, t}\left(\theta_{i}\right)$.

Figure 2 shows realization of scoring factors for different errors and confidence level. From the graph presented in Figure 2, it is import to notice that,

- when a CA is highly confident about its prediction $\left(e . g \sigma_{i, t}=0\right)$; then highest score is rewarded i.e when the realized absolute error is zero.

- when the realized error is relatively higher, then CA will be benefitted to report lower confidence (e.g higher values of $\sigma_{i, t}$ ).

Therefore, CAs can maximize the score to report the uncertainty of prediction accurately.

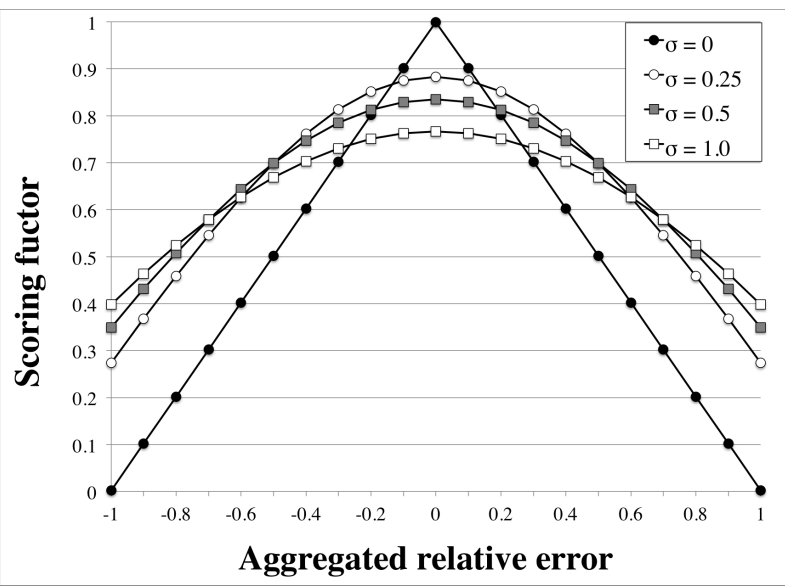

Fig. 2. A CRPS scoring mechanism for different errors

\section{Step3: Bids task candidates}

CAs bid for the task candidates to CDRS. In this model, a single task means that CA shifts a "flexible load" from specific $t_{h}$ to specific $t_{l}$. A bid consists of four elements as presented in Table 1. In this paper, each CA selects $t_{h}$ and $t_{l}$ randomly. Each CA can bid a defined number in advance.

A bid consists of three elements, beginning of shift demand $t_{h}$, end of shift demand $t_{l}$, shift demand capacity $s d_{i, t}^{c}$. In this paper, each CA selects $t_{h}$ and $t_{l}$ randomly. Each CA can bid a defined number in advance.

\section{Step4: Accepts based on threshold}

CDRS accepts bids from CAs based on threshold. First, CDRS sorts all bids based on $s d_{i, t}^{p} *\left(1-\sigma_{i, t}\right)$. That is, larger the contribution of peak cut, higher is the accept rate. Next, CDRS accepts bids based on following constraints in Eq.(8) and Eq.(9).

[Conditions of Acceptance]

$$
\begin{aligned}
& \text { TotalDemand }_{t_{h}}-\sum_{b \in B i d s_{t}} s d_{b, t}^{p} \geqslant T h \\
& \text { TotalDemand }_{t_{l}}+\sum_{b \in B i d s_{t}} s d_{b, t}^{p}<T h
\end{aligned}
$$

where Bids $s_{t}$ means sorted all bids. Eq.(8) shows that total demand in $t_{h}$ is always larger than threshold. That is, this model can not conduct peak 
cut more than necessary in order to guarantee the safety. Eq.(9) shows that total demand in $t_{l}$ is always smaller than threshold. That is, this model can not create new peak demand.

\section{Step5: Reports aggregated prediction}

CDRS aggregates the prediction from CAs and reports it to GENCO. The task of achieving the prediction and actuality can be expressed as $s d_{c, t}^{p}=$ $\sum_{i \in N} s d_{i, t}^{p}, s d_{c, t}^{a}=\sum_{i \in N} s d_{i, t}^{a}$, respectively. The relative error and confidence level of CDRS can be expressed as, $e_{c, t}=\frac{s d_{c, t}^{a}-s d_{c, t}^{p}}{s d_{c, t}^{p}}, \sigma_{c, t}^{2}=\frac{\sum_{i \in N}\left(s d_{i, t}^{p} * \sigma_{i, t}\right)^{2}}{\left(\sum_{i \in N} s d_{i, t}^{p}\right)^{2}}$, respectively. Therefore, the as the number of CA increases, the relative error of CDRS decreases. In the experiment, we show that, as the number of $\mathrm{CA}$ increases, prediction accuracy increases.

\section{Step6: Calculates the profit}

GENCO calculates the profit based on production cost and actual shift demand of CAs. The GENCOs usually operate multiple plants of different types, e.g. gas, hydroelectric (hydro), renewables and coal. These plants may be categorized as base, intermediate, and peak-load. The base-load plants generally have a higher capital cost but low operating cost, and thus run all of the time (e.g., hydro, nuclear). Intermediate load plants (e.g., coal) have a higher operating cost, and peak load plants (e.g., gas turbines) have the highest operating cost. In any given time periods, if CDRS demand exceeds the base-load capacity, the generator turns to the intermediate-load plants and then finally, to peak-load plants to generate additional electricity. Lets $c_{h}$ and $c_{l}$ represent the peak-load production cost in $t_{h}$ and intermediate production cost in $t_{l}$ respectively and the difference $c_{h}-c_{l}$ represents the costValue , the profit of GENCO is presented in Eq.(10).

$$
\operatorname{profit}_{t}^{G}=\operatorname{costValue}_{t} \times s d_{c, t}^{a}
$$

In this paper, the production cost is based on real data ${ }^{11,29}$.

\section{Step7: Calculates the revenue}

GENCO calculates the payment based on prediction and confidence of CDRS's task achievement. Let the price difference $p_{h}-p_{l}$ represents the $s v_{t}$, the payment is presented in Eq.(11).

$$
\begin{aligned}
& v_{c, t}(a, \theta)= \\
& \operatorname{CRPS}_{c, t}(\theta) \times s v_{t} \times s d_{c, t}^{a}+\lambda \times \operatorname{profit}_{t}^{G}(11)
\end{aligned}
$$

The payment is composed of four factors. The CRPS (scaled between 0 and 1) is the "accuracy factor" that incentivizes CDRS to provide as accurate description as possible for its relative prediction error. The $s v_{t}$ is the "shift factor" that constitutes the actual price paid by the GENCO to CDRS. The $s d_{c, t}^{a}$ is the actual shift demand of CDRS in settlement time period $t$, which is independently observed by the GENCO. The $\lambda \times$ profit $(0<\lambda \leqslant 1)$ is the fraction of production profit. Since GENCO needs to make profits by CDR, GENCO allocates a fraction of production profit. The significant point of Eq.(11) is that it considers not only the different price, but also incentive to provide accurate description. Therefore, our model can remove the "unethical" ways such as falsehood and guarantee the safety.

\section{Step8: Allocates the revenue}

CDRS allocates the payment to CAs based on contribution rate in the total reward and part of GENCO's profit. The contribution rate is composition of actual shift demand and prediction of accuracy. The reward of $C A_{i}$ is presented in Eq.(12).

$$
\begin{gathered}
v_{i, t}\left(a, \theta_{i}\right)=\frac{\operatorname{CRPS}_{i, t}\left(\theta_{i}\right) \times s d_{i, t}^{a}}{\sum_{j \in N} \operatorname{CRPS}_{j, t}\left(\theta_{j}\right) \times s d_{j, t}^{a}} \times v_{c, t}(a, \theta) \\
\text { where } v_{c, t}(a, \theta) \text { shows the Eq.(11). }
\end{gathered}
$$

\section{Algorithm properties}

Proposed algorithms have several desirable properties. 


\section{Truthfulness}

Truthfulness is an important measure to validate an agent based protocol or strategy. One obvious way to deal with truthfulness is to set a mediator which will observe the behavior of all participated agents and thus ensures all agents report truthfully. However, mediator based protocols impose several difficulties such as centralization, private information elicitation, etc. At the same time, as the number of agent increases, Then it will become difficult for a single mediator to control the information. Therefore, the best way to ensure is to design the protocol such a way that, its always the best for an agent to report truthfully in order to get maximum expected utility. Our model is strategy-proof by using VCG mechanism and Scoring rule.

Theorem 1. CDR is strategy-proof for CAs with quasi-linear preferences.

Proof. We prove that CDR is strategy-proof, such that truth-revelation is a dominant strategy for each $\mathrm{CA}$, from which allocative efficiency follows immediately because the choice rule $g(\theta)$ computes the efficient allocation in Eq.(1).

The utility to CA $i$ from strategy $\widehat{\theta}_{i}$ is:

$$
\begin{aligned}
& u_{i}\left(\widehat{\boldsymbol{\theta}}_{i}\right) \\
& =v_{i}\left(g(\widehat{\boldsymbol{\theta}}), \theta_{i}\right)-p_{i}(\widehat{\boldsymbol{\theta}}) \\
& =v_{i}\left(g(\widehat{\boldsymbol{\theta}}), \theta_{i}\right)+\sum_{j \neq i} v_{j}\left(g(\widehat{\boldsymbol{\theta}}), \widehat{\boldsymbol{\theta}_{j}}\right)-\sum_{j \neq i} v_{j}\left(g\left(\widehat{\boldsymbol{\theta}_{-i}}\right), \widehat{\boldsymbol{\theta}_{j}}\right)
\end{aligned}
$$

Ignoring the final term, because $\sum_{j \neq i} v_{j}\left(g\left(\theta_{-i}\right), \theta_{j}\right)$ is independent of an CA $i$ 's reported type.

we prove that truth-revelation $\widehat{\theta}_{i}=\theta_{i}$ solves:

$$
\begin{aligned}
& \max _{\widehat{\theta}_{i} \in \Theta_{i}}\left[v_{i}\left(g\left(\widehat{\theta}_{i}, \widehat{\theta_{-i}}\right), \theta_{i}\right)+\sum_{j \neq i} v_{j}\left(g\left(\widehat{\theta_{i}}, \widehat{\theta_{-i}}\right), \theta_{j}\right)\right] \\
& =\max _{\widehat{\theta}_{i} \in \Theta_{i}}\left[v_{i}\left(x, \theta_{i}\right)+\sum_{j \neq i} v_{j}\left(x, \widehat{\theta_{j}}\right)\right]
\end{aligned}
$$

where $x=g\left(\widehat{\theta_{i}}, \widehat{\theta_{-i}}\right)$ is the outcome selected by the mechanism. The only effect of the CA's announced type $\widehat{\theta}_{i}$ is on $x$, and the CA can maximize Eq.(13) by announcing $\widehat{\theta}_{i}=\theta_{i}$ because $v_{i}$ is strictly proper scoring rule in Eq.(11) and Eq.(12). As shown Eq.(11), the CRPS part of function is a strictly proper scoring rule and the entire function in Eq.(11) is an affine transformation of this rule (since it only involves multiplication and addition with other factors which do not depend on the reports made by the agent). Hence, Eq.(11) and Eq.(12) are also strictly proper ${ }^{15}$. Then the mechanism computes $g\left(\widehat{\theta}_{i}, \widehat{\theta_{-i}}\right)$ to explicitly solve:

$$
\begin{aligned}
& \max _{a \in A} v_{i}\left(a, \theta_{i}\right)+\sum_{j \neq i} v_{j}\left(a, \widehat{\theta}_{j}\right) \\
& =\max _{a \in A} \frac{C R P S_{i, t}\left(\theta_{i}\right) \times s d_{i, t}^{a}}{\sum_{j \in N} \operatorname{CRPS} S_{j, t}\left(\theta_{j}\right) \times s d_{j, t}^{a}} \times v_{c, t}+\sum_{j \neq i} v_{j}\left(a, \widehat{\theta}_{j}\right)
\end{aligned}
$$

Truth-revelation is the dominant strategy of CA $i$, whatever the reported types $\widehat{\theta_{-i}}$ of the other CAs.

\section{Individual rationality}

Individual rationality is ascertained for all CAs in CDRS, as they all have non-negative expected gain from participation. Our model is individualrationality by using VCG mechanism.

Theorem 2. CDR is individual-rational with quasilinear preferences.

Proof. To show individual-rationality, we show that the utility to CA $i$ in the equilibrium outcome of the mechanism is always non-negative. We can assume truth-revelation in equilibrium. The utility to CA $i$ with type $\theta_{i}$ is:

$$
\begin{aligned}
& u_{i}\left(\theta_{i}, \theta_{-i}\right) \\
& =v_{i}\left(g(\theta), \theta_{i}\right)-\left(\sum_{j \neq i} v_{j}\left(g\left(\theta_{-i}\right), \theta_{j}\right)-\sum_{j \neq i} v_{j}\left(g(\theta), \theta_{j}\right)\right) \\
& =\sum_{i} v_{i}\left(g(\theta), \theta_{i}\right)-\sum_{j \neq i} v_{j}\left(g\left(\theta_{-i}\right), \theta_{j}\right)
\end{aligned}
$$

Eq.(14) is actually the same as VCG mechanism. Hence, the equilibrium outcome of the mechanism is always non-negative. 
Eq.(14) is non-negative because the value of the best solution without CA $i, \sum_{j \neq i} v_{j}\left(g\left(\theta_{-i}\right), \theta_{j}\right)$, cannot be greater than the value of the best solution with CA $i, \sum_{i} v_{i}\left(g(\theta), \theta_{i}\right)$. This follows because any choice with agents $j \neq i$ is also feasible with all CAs (monotonicity), and has just as much total value (no negative externalities).

\section{Scalability}

Scalability is important property in the field of smart grid because there would be so many participants. In our model, the reward is based on actual demand shift and accuracy of prediction using CRPS. We assume that each CA is various environments such as communication speed and load composition. Therefore, the more number of CA increase, Wisdom of Crowds ${ }^{27}$ can work and the accuracy of prediction of CDRS increase. We show that the large number of CA are better accuracy of prediction in experiment.

\section{Experimental Results}

\subsection{Setting}

In this section, some data analysis and simulation results are presented in order to verify the feasibility of the scoring rule based cooperative demand response mechanism. The real parameters are taken based on Chubu Electric Power Co.,Inc., such as the demand curve ${ }^{10,28}$ and production cost ${ }^{11,29}$. We conducted 62 days in summer. In each day, we applied a CDR mechanism. The demand prior to CDR is based on above data and perturbed up to $10 \%$ by a uniformly distributed random number.

We compared our model with the singleton algorithm in Figure 3. CAs report prediction to GENCO without CDRS and calculate the reward based on Eq.(11). The parameters for our experiments are defined as Table 2.

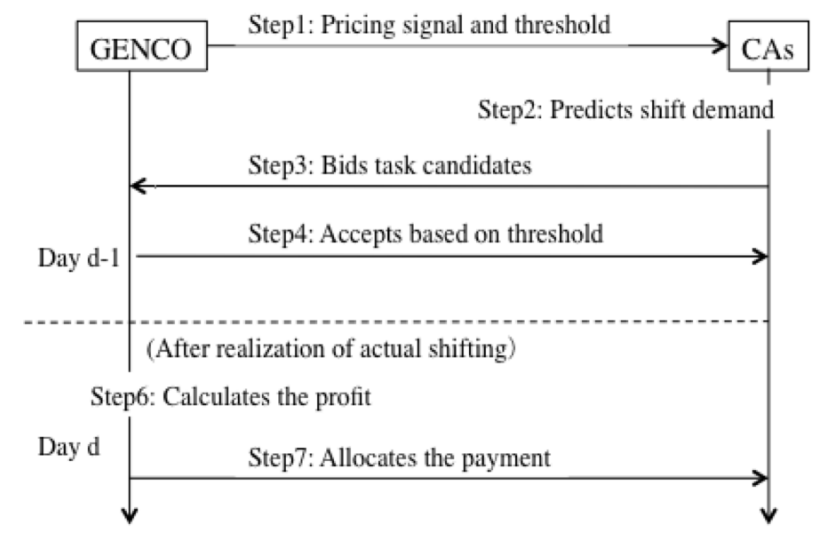

Fig. 3. Singleton model flow

\subsection{Results}

While the quantitative results of these simulations will vary from market to market, the qualitative results suggest that CDR mechanism can indeed help GENCO and CAs.

\section{Effectiveness of peak cut}

Let us first consider the effectiveness in terms of peak cut during half of simulation days. In Figure 4 , the vertical axis represents the total energy consumption and the horizontal axis represents the half of simulation hour. We can see that before-demandcurve has the cyclic electricity consumption pattern based on real data and the peak demands of afterdemand-curve are always reduced. Therefore, scoring rule and VCG based proposed mechanism is capable to reduce peak demand.

Figure 5 shows the total demand curve and threshold. In Figure 5, $t_{10}, t_{11}, t_{12}, t_{14}, t_{15}, t_{16}, t_{17}, t_{20}$ belong to $t_{h}$, other belong to $t_{l}$. Before-peak-demand is $1292.4 \mathrm{kwh}$, threshold is $1163.1 \mathrm{kwh}$, after peak demand is $1218.9 \mathrm{kwh}$. We can see that proposed mechanism can flatten peak demand without creating a new peak. 


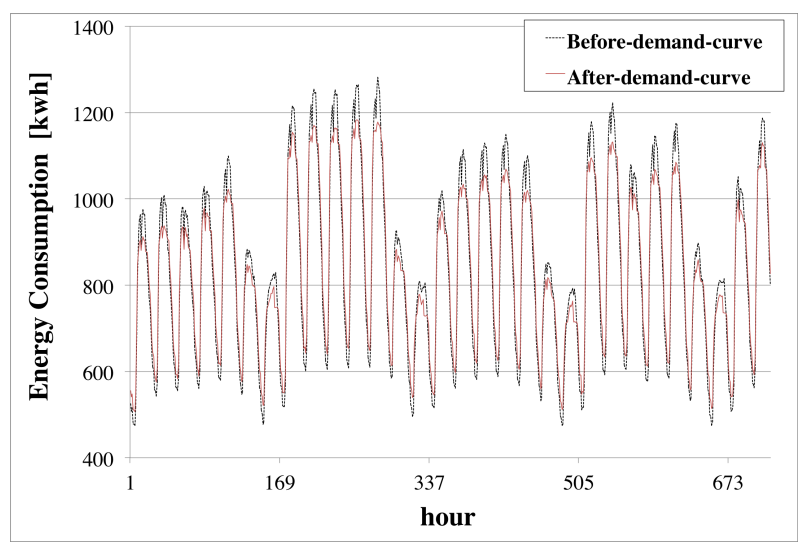

Fig. 4. Energy usage before (black dotted line) and after (red solid line) using of CDR algorithm: 31 days

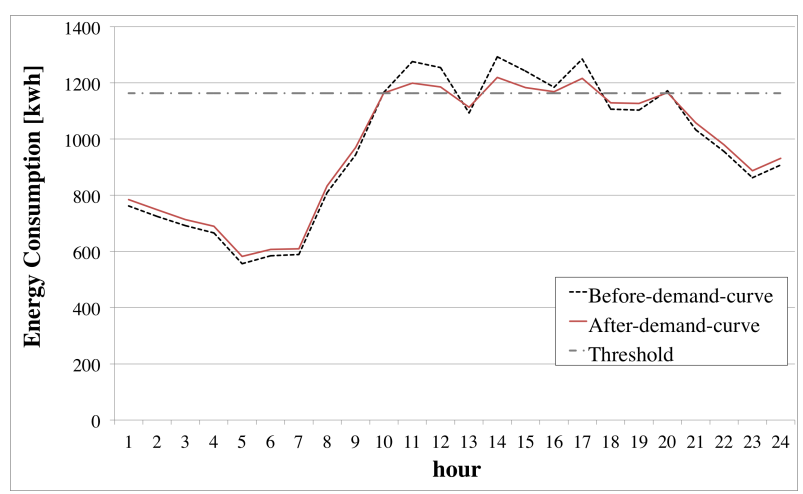

Fig. 5. Energy usage before (black dotted line) and after (red solid line) using of CDR algorithm: 24 hours

\section{CA's average utility: CDRS vs Singleton}

Figure 6 shows the CA's average utility (total reward divide by number of CA). In Figure 6, the vertical axis represents the CA's average utility in a day and the horizontal axis represents the total simulation days. We can see that CDRS is always better reward than Singleton. That is, each CA has incentive to cooperate with each other because each $\mathrm{CA}$ can increase the utility.

We can see that the utility of CDRS and Singleton are always non-negative. This is because we apply VCG mechanism to our mechanism and individual-rationality is ascertained for all CAs.

\section{Scalability: the large number of CA}

Table 3 shows scalability of our mechanism considering number of CA and CA's average reward during total simulation days. AVE in the table represents the CA's average utility. We can see that the more number of CA increases, the more increases average utility both CDRS and Singleton. However, the member of CDRS is better than Singleton because the more number of CA increase, the better of prediction accuracy and also utility.

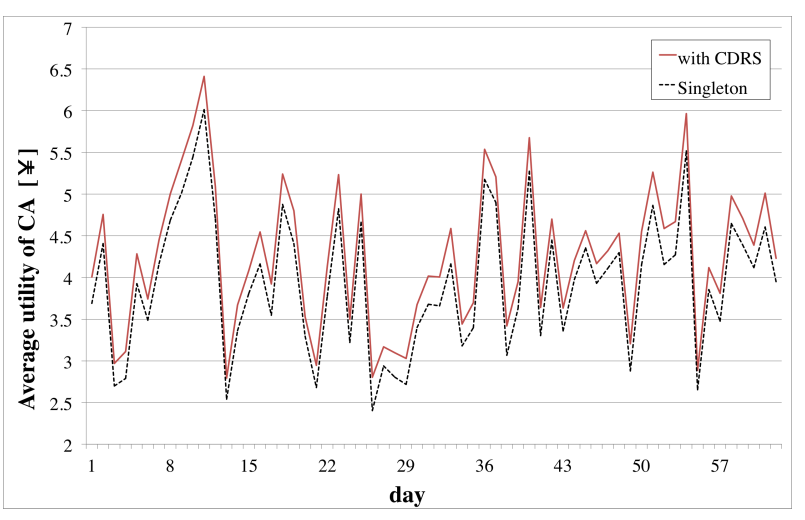

Fig. 6. CA's average utility with CDRS vs Singleton

\section{Related Work}

In the field of DR, there have been several works that focused on scoring rule ${ }^{24,7} .{ }^{24}$ presented a methodology for predicting aggregated demand in smartgrid. But these method do not considers demand shift at the device level and do not examine these method by real data simulation. Our model can take heterogeneity at the device level into account and the proposed mechanism is tested on real data.

${ }^{7}$ presented a pricing scheme for smart house environment which takes advantage of both time and incentive based DR using CRPS. But this scheme do not considers the conditions of the demand shift and do not considers the payment to mechanism. Our model can consider the conditions of the demand shift and we combine scoring rule and VCG mechanism to truthfully elicit agent's private preferences.

In addition, most studies $13,12,20,7$ have not focused on cooperation among consumers although it is important to devise an efficient and stable DR. 
Our model focused on cooperation among consumer agents using CRPS.

There have been a few studies that focused on cooperation among agents. ${ }^{14}$ presented a formulation for scheduling demand response among residences when the cost of electricity is known in advance. They deal with cooperation as a utility optimization problem. But they do not consider prediction of demand and scalability of method. ${ }^{22}$ focused on demand side management in terms of Virtual Power Plant and they deal with cooperation using market mechanism. But they do not consider demand shift at the device level.

On the other hand, our model achieves the cooperation using scoring rule and develop some of the desired properties such as truthfulness and individual rationality and scalability by applying CRPS and VCG mechanism to our model. In addition, our model can consider demand shift at the device level using bid.

\section{Conclusion}

DR program is gaining importance in a smart grid environment. Due to the lack of energy supply in comparison with demand, efficient energy management and a DR mechanism is essential. This paper introduces a scoring rule based truthful demand response mechanism considering both pricing factor and incentive factor.

In our model, some of the desired properties such as truthfulness and individual-rationality are proved. We fulfill those properties by combining scoring rule and VCG mechanism. In addition, our model is that each CA has incentive to participate in CDR using Wisdom of Crowds such as a lot of CAs prediction is better than a CA prediction. This model consists of generators, cooperative demand response system and consumers. The scoring system (facilitating Continuous Ranked Probability Score) and VCG mechanism are designed such a way that it will incentivize the consumer agents to predict accurately. In the experimental results, we presented that this mechanism is capable to reduce energy consumption and mechanism is scalable.

We will try to model device sensitiveness to- wards scheduling and apply such mechanism for higher scaled power system. Moreover, another potential future research direction is to integrate device dependency in formulation to make the DR program responding towards the heterogeneity of devices. Moreover, we will try to optimize the threshold to flatten peak demand.

1. M.-R. A.-H. and L.-G. A. Optimal residential load control with price prediction in real-time electricity pricing environments. In IEEE Transactions on Smart Grid, 1:120-133, 2010.

2. M. Albadi and E. El-Saadany. A summary of demand response in electricity markets. Electric Power Systems Research, 78:1989-1996, 2008.

3. S. Borenstein. The long-run efficiency of real-time electricity pricing. The Energy Journal, 26(3):93-116, 2005.

4. C. Boutilier. Eliciting forecasts from self-interested experts: scoring rules for decision makers. In In Proceedings of the 11th International Conference on Autonomous Agents and Multi-Agent Systems (AAMAS2012), 2012.

5. G. Brier. Verification of forecasts expreseed in terms of probability. Monthly Weather Review, 78(1):1-3, 1950.

6. S. Caron and G. Kesidis. Incentive-based energy consumption scheduling algorithms for the smart grid. In IEEE International Conference on Smart Grid Communications, pages 391-396, 2010.

7. S. Chakraborty, T. Ito., and T. Senju. Smart pricing scheme: A multi-layered scoring rule application. Expert Systems with Applications, 41:3726-3735, 2014.

8. L. Chen, S. L. N. Li, and J. Doyle. Two market models for demand response in power networks. In IEEE International Conference on Smart Grid Communications, pages 397-402, 2010.

9. E. H. Clarke. Multipart pricing of public goods. Public Choice, 11, 1971.

10. C. E. P. Company. electricity demand performance (2013). 2013.

11. C. E. P. Company. Relationship between power resources and usage of electricity in a day. 2013.

12. M. Doostizadeh and H. Ghasemi. A day-ahead electricity pricing model based on smart metering and demand-side management. Energy, 46:221-230, 2012.

13. P. Du and N. Lu. Appliance commitment for household load scheduling. IEEE Trans. Smart Grid, 2(2):411-419, 2011.

14. N. Gatsis and G. B. Giannakis. Cooperative multiresidence demand response scheduling. Information Sciences and Systems (CISS), 2011.

15. T. Gneiting and A. Raftery. Strictly proper scoring 
rules, prediction and estimation. Journal of the American Statistical Association, 102:359-378, 2007.

16. T. Groves. Incentives in teams. Econometrica, 41(4), 1973.

17. S. Holland and E. Mansur. Is real-time pricing green? the environmental impacts of electricity demand variance. Rev. Econ. Stat., 90(3):550-561, 2008.

18. S. Holland and E. Mansur. Is real-time pricing green? the environmental impacts of electricity demand variance. Rev. Econ. Stat., 90(3):550-561, 2008.

19. E. Ianovski, L. Yu, E. Elkind, and M. C. Wilson. The complexity of safe manipulation under scoring rules. In In Proceedings of the Twenty-First International Joint Conference on Artificial Intelligence (IJCAI-11), 2011.

20. D. Li, S. Jayaweera, and A. Naseri. Auctioning game based demand response scheduling in smart grid. IEEE Online Conference on Green Communications, pages 58-63, 2011.

21. N. Li, L. Chen, and S. Low. Optimal demand response based on utility maximization in power networks. IEEE Power and Energy Society General Meeting, 2011.

22. K. Ramachandra, C. Georgios, R. Valentin, R. Alex, and J. N. R. Cooperatives for demand side management. The Seventh Conference on Prestigious Applications of Intelligent Systems, 2012.

23. V. Robu, R. Kota, G. Chalkiadakis, A. Rogers, and N. R. Jennings. Cooperative virtual power plant formation using scoring rules. In In Proc. of the 26th Conference on Artificial Intelligence (AAAI-12), pages
370-376, 2012.

24. H. Rose, A. Rogers, and E. H. Gerding. A scoring rule-based mechanism for aggregate demand prediction in the smart grid. In In Proceedings of the 11th International Conference on Autonomous Agents and Multi-Agent Systems (AAMAS-2012), 2012.

25. P. Samadi, A. Mohsenian-Rad, R. Schober, and V. W. S. Wong. Advanced demand side management for the future smart grid using mechanism design. IEEE Transaction on Smart Grid, 1(3), 2012.

26. P. Samadi, A. Mohsenian-Rad, R. Schober, V. W. S. Wong, and J. Jatskevich. Optimal real-time pricing algorithm based on utility maximization for smart grid. In IEEE Smart Grid Communications, pages 415-420, 2010.

27. J. Surowiecki. The Wisdom of Crowds: Why the Many are Smarter Than the Few and how Collective Wisdom Shapes Business, Economies, Societies, and Nations. Doubleday, 2004.

28. the Agency of Natural Resources and Energy. Demand structure estimation of a summer maximum dissipation use day. 2011.

29. N. P. Unit. Verification committee's reports in terms of cost. 2011.

30. W. Vickrey. Counterspeculation, auctions, and competitive sealed tenders. The Journal of Finance, 16(1), 1961.

31. L. Xia and V. Conitzer. Finite local consistency characterizes generalized scoring rules. In In Proceedings of the Twenty-First International Joint Conference on Artificial Intelligence (IJCAI-09), 2009. 